\title{
Adaptive Subcarrier Modulation for Indoor Public Safety Body-to-Body Networks
}

\author{
Thijs Castel $^{1}$, Sam Lemey ${ }^{1}$, Sam Agneessens ${ }^{1}$, Patrick Van Torre ${ }^{1}$, Hendrik Rogier ${ }^{1}$, Claude Oestges ${ }^{2}$ \\ ${ }^{1}$ Department of Infomation Technology, Ghent University/iMinds, Sint-Pietersnieuwstraat 41, B-9000 Ghent, Belgium \\ ${ }^{2}$ Institute of Information and Communication Technologies, Electronics and Applied Mathematics, \\ Université Catholique de Louvain, Place du Levant 3, B-1348 Louvain-la-Neuve, Belgium
}

\begin{abstract}
In this contribution, we present the Bit Error Rate characteristics for an indoor, wideband body-to-body channel between two firefighters when using IEEE 802.11 ac, which is proven a very suitable standard for future, wideband public safety networks. Moreover, the BER and throughput characteristics, when applying both transmission blocking, fixed and adaptive, subcarrier modulation are presented. These characteristics show an increased throughput when applying adaptive subcarrier modulation. We have conducted a wideband, indoor channel sounder campaign at $3.6 \mathrm{GHz}$ with $120 \mathrm{MHz}$ useful bandwidth, simulating real-life rescue operations performed by two simultaneously moving members of the Rapid Intervention Team. Both firefighters were equipped with low-profile, lightweight and energy-efficient Ultra Wideband Cavity-Backed slot antennas in Substrate Integrated Waveguide technology, unobtrusively deployed inside the front and back sections of their jackets, providing $2 \times 2$ MIMO capability.
\end{abstract}

Index Terms-Body-to-Body, Textile antenna, Public Safety Networks, Adaptive Subcarrier Modulation

\section{INTRODUCTION}

Future Public Safety Networks, which support communication between on-duty firefighters, are expected to evolve from narrowband towards wideband Wireless Body Area Networks (WBANs) [1], [2]. By applying Orthogonal Frequency Division Multiplexing (OFDM), these wideband, frequencyselective channels can be subdivided into multiple frequencyflat, orthogonal subcarriers, increasing the total throughput compared to state-of-the-art narrowband public safety networks. Moreover, the highest possible modulation order, which still guarantees a sufficiently low Bit Error Rate (BER), may be applied per frequency-flat subcarrier. This strategy increases the throughput on each subcarrier, and hence, maximizes the total throughput. This high throughput, combined with a low BER, allows multimedia broadcast and efficient communication of real-time on-body sensor data between on-duty firefighters. Moreover, the rescue workers' situational awareness increases whereas the response time of operation decreases, which is a major advantage in indoor public safety networks where minutes, or even seconds count.

Therefore, a wideband indoor body-to-body channel sounder campaign, replicating real-life rescue operations performed by the Rapid Intervention Team (RIT), has been carried out in an office environment. Both members of the RIT, typically operating in each others vicinity, were equipped with two lowprofile, light-weight Ultra-Wideband (UWB) textile antennas
[3], unobtrusively integrated in the front and back sections of their firefighter jackets, providing a $2 \times 2$ MIMO link.

Considering that the IEEE 802.11 ac standard [4] is proven very suitable for indoor body-to-body channels, we analyze the BER performance of the four modulations supported by this wideband standard, being 4-, 16-, 64- and 256 Quadrature Amplitude Modulation (QAM). Moreover, we prove that the 2x2 MIMO wideband, body-to-body channel can be subdivided into two independent, quasi-uncorrelated spatial subchannels which simultaneously transmit data, providing multiplexing, and, hence, throughput gain. The throughput of these two spatial subchannels is further optimized by fixed or adaptive subcarrier modulation with transmission blocking [5]. This optimization algorithm blocks transmission on subcarriers leading to a BER higher than the pre-set BER threshold while assigning the same, or an adaptively changing, modulation to the other, active subcarriers that guarantee a BER smaller than or equal to the pre-set BER threshold.

A simple adaptive modulation technique for individual subcarriers was already proposed in 1996 by Czylwik [6], showing that the required power can be dramatically reduced when applying adaptive, instead of fixed, subcarrier modulation. Through the years, more complex adaptive modulation schemes were proposed, focussing on trade-off between performance and throughput [7], maximizing spectral efficiency [8] or minimizing transmission energy [9]. However, only [10] proposes an adaptive scheme for the WBAN physical layer, concentrating on off-body communication. Increasing BER performance and decreasing possible are demonstrated. This paper proposes, for the first time in literature, an analysis of fixed and adaptive subcarrier modulation with transmission blocking for indoor, wideband body-to-body channels. The scheme maximizes the potential throughput and, hence, data rate in future wideband public safety networks.

\section{Measurement Setup}

A real-life rescue operation, performed by the Rapid Intervention Team looking for potential victims, was replicated by mobile measurements during which both firefighters were simultaneously moving around on the same floor of an office block, as shown in Fig. 1. Both the TX and RX firefighters were equipped with two low-profile, light weight and flexible Ultra Wideband cavity-backed slot antennas in Substrate Integrated 


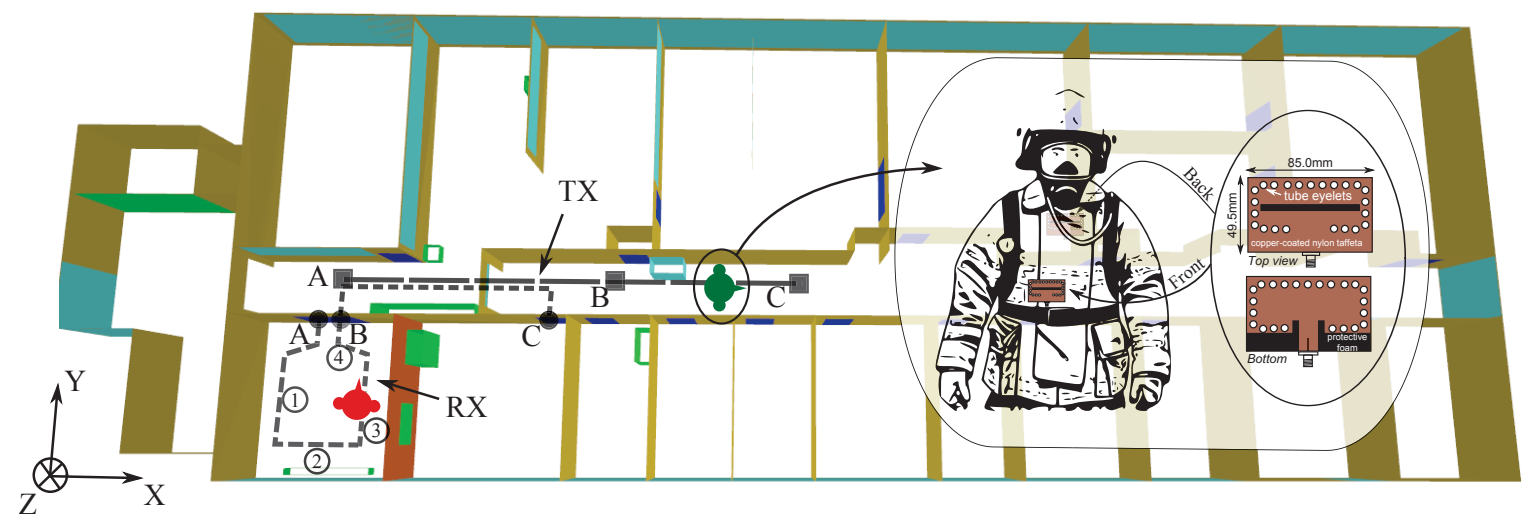

Fig. 1. Simplified indoor office model with the wideband body-to-body measurement scenario together with the front and back locations of the two integrated UWB SIW textile antennas, for both the TX and RX firefighter

Waveguide (SIW) technology [3]. These antennas provide stable radiation characteristics when placed on different on-body locations. Moreover, they are unobtrusively integrated inside the front and back sections of the firefighter jackets, as shown in Fig. 1, implementing $2 \times 2 \mathrm{MIMO}$. When both firefighters enter a building, the RX firefighter, whose trajectory is marked by the short dashed line on Fig. 1, starts scanning the offices whereas the TX firefighter, whose trajectory is marked by the longer dashed line, is simultaneously scanning the hallway, while he remains close to the RX firefighter. The markers A, B and $\mathrm{C}$, placed along both firefighter trajectories, indicate where the firefighters are located at the same time instant during the one-minute-long measurement, collecting 4650 measurement cycles for all four body-to-body links. Measurements were performed using the Elektrobit channel sounder at $3.6 \mathrm{GHz}$ center frequency with $120 \mathrm{MHz}$ useful bandwidth. The TX power was chosen equal to $20 \mathrm{dBm}$ to obtain reliable wideband, body-to-body channel measurements.

\section{MIMO-OFDM}

\section{A. Compatibility with the IEEE 802.11 ac standard}

By means of the high-resolution power delay profiles, directly provided by the Elektrobit channel sounder, the maximum RMS delay spread during $90 \%$ of the time, defined as $\tau_{R M S, 90}$ is found for the Front to Back (F2B) link. We obtain $\tau_{R M S, 90}$ equal to $45.80 \mathrm{~ns}$. The minimal $50 \%$ correlation bandwidth during $90 \%$ of the time, defined as $B_{C, 0.5,90}$, equals $3.92 \mathrm{MHz}$ for the F2B link [11]. When employing OFDM, the wideband, frequency-selective, body-to-body channel is subdivided into $N$ frequency-flat, lower data rate, orthogonal subcarriers. These modulated subcarriers experience frequency flat fading when their subcarrier bandwidth is smaller than one tenth of the minimal 50\% correlation bandwidth [11]. When focusing on the time domain, a Cyclic Prefix (CP) is added in between two consecutive OFDM symbols on one subcarrier to avoid Inter Symbol Interference (ISI). The minimal CP length should be larger than, or equal to, 3 times the maximal RMS delay spread [12]. These calculated OFDM parameters are compatible with the IEEE 802.11 ac standard, which sets the minimal cyclic prefix length equal to 400 ns seconds, being larger than 137.4 $\mathrm{ns}$, and the subcarrier bandwidth equal to $312.5 \mathrm{kHz}$, being smaller than $392 \mathrm{kHz}$. For further calculations, the wideband indoor body-to-body channel is subdivided into 256 frequencyflat subcarriers, corresponding to a total OFDM bandwidth equal to $80 \mathrm{MHz}$.

\section{B. Eigenvalue Decomposition}

When employing MIMO-OFDM, the MIMO channel can be subdivided into $r$ equivalent SISO spatial subchannels, with $r=$ $\min \left(N_{T X}, N_{R X}\right)$. The received Signal to Noise Ratio (SNR) of these SISO spatial subchannels is calculated via the eigenvalue decomposition of $\mathbf{H}_{\mathbf{k}} \cdot \mathbf{H}_{\mathbf{k}}{ }^{H}$, with $\mathbf{H}_{\mathbf{k}}$ the channel matrix of subcarrier $k$. This leads to a column vector of eigenvalues $\lambda_{\mathbf{k}}=\left[\begin{array}{llll}\lambda_{1, k} & \lambda_{2, k} & \ldots & \lambda_{r, k}\end{array}\right]^{T}$. The received SNR on subcarrier $k$ for spatial subchannel $i$ is than calculated as:

$$
\mathrm{SNR}_{i, k}=\frac{P_{k}}{N_{T X} \cdot \sigma^{2}} \cdot \lambda_{i, k}
$$

with $P_{k}$ the transmit power allocated to subcarrier $k, \sigma^{2}$ the noise power and $N_{T X}$ the number of transmit antennas. The eigenvalue decomposition, dependent on the spatial correlation between the links from any transmit to any receive antenna, gives an indication of the possible multiplexing gain, per subcarrier, when applying MIMO-OFDM. This implies that the spatial multiplexing gain per subcarrier, when applying $2 \times 2$ MIMO, is largest if the two eigenvalues are equal, corresponding to fully uncorrelated, equal-gain, spatial channels, and hence fully exploiting parallelism. When using the method described in [13], the spatial correlation between the F2F and B2B link is found equal to 0.17 . This indicates that the channel coefficients are sufficiently decorrelated to ensure spatial multiplexing, and hence, throughput gain per subcarrier when subdividing the $2 \times 2$ MIMO-OFDM channel into two independent, quasiuncorrelated spatial subchannels.

\section{Bit Error Rate}

Define $M$ the number of constellation points and $Q$ the Q-function such that, when employing Gray-mapping, the Bit 
Error Rate of subcarrier $k$ for spatial subchannel $i$ is calculated as:

$$
\mathrm{BER}_{i, k}=\frac{N_{b}}{\log _{2}(M)} Q\left(\sqrt{\frac{d_{m i n}^{2}}{2} \cdot \mathrm{SNR}_{i, k}}\right),
$$

with:

$$
\begin{gathered}
N_{b}=\frac{4(\sqrt{M}-1)}{\sqrt{M}}, \\
d_{\text {min }}^{2}=\frac{6}{M-1} .
\end{gathered}
$$

Define $L$ the number of measurement cycles, $r$ the number of spatial subchannels and $N$ the number of frequency-flat subcarriers such that the mean received SNR and mean BER are calculated as:

$$
\begin{aligned}
& \overline{\mathrm{SNR}}=\frac{1}{L} \sum_{l=1}^{L}\left(\frac{1}{r} \sum_{i=1}^{r}\left(\frac{1}{N} \sum_{k=1}^{N} \mathrm{SNR}_{l, i, k}\right)\right) \\
& \overline{\mathrm{BER}}=\frac{1}{L} \sum_{l=1}^{L}\left(\frac{1}{r} \sum_{i=1}^{r}\left(\frac{1}{N} \sum_{k=1}^{N} \mathrm{BER}_{l, i, k}\right)\right)
\end{aligned}
$$

Fig. 2 presents the BER characteristic of the different modulations supported by the IEEE 802.11 ac standard when employing uniform power allocation. Due to body shadowing effects, 4QAM performs worse than the simulated 4QAM curve for Rayleigh fading and diversity order 1 . Also note that for the same $\overline{\mathrm{SNR}}$, the higher order modulations lead to higher $\overline{\mathrm{BER}}$ due to the reduced noise margin.

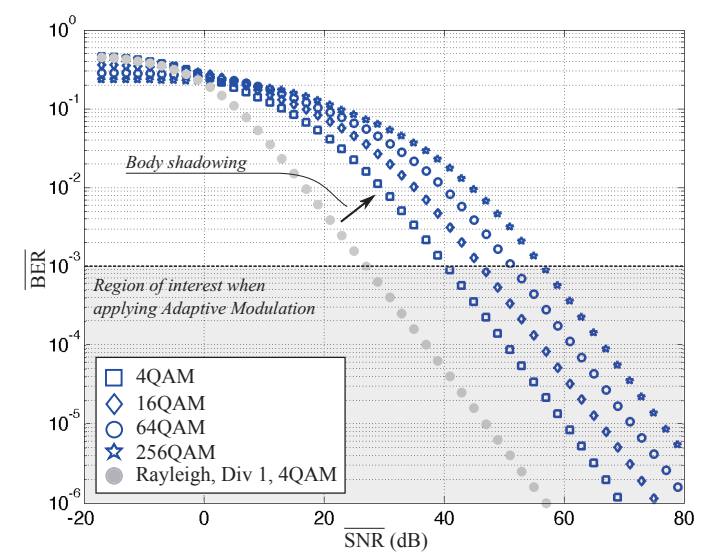

Fig. 2. Mean BER performance for two spatial subchannels, of all four modulations supported by the IEEE 802.11 ac standard

\section{TRANSMISSION BLOCKING MODULATION}

\section{A. Transmission blocking fixed subcarrier modulation}

In this Section, we analyze the performance of a wireless body-to-body system which uses perfect Channel State Information (CSI) to ensure a $\overline{\mathrm{BER}} \leq \mathrm{BER}_{\text {thresh. }}$. By means of channel feedback, transmission blocking is applied on subcarriers which experience a $\mathrm{SNR}<\mathrm{SNR}_{\text {thresh }}$, leading to a BER $>\mathrm{BER}_{\text {thresh }}$. These non-used subcarriers are "turned of", without subcarrier power reallocation, so that the SNR and BER on these non-used subcarriers are not defined whereas the throughput is set to 0 bits/symbol. In contrast, all subcarriers who experience a SNR $\geq \mathrm{SNR}_{\text {thresh }}$ leading to $\mathrm{BER} \leq \mathrm{BER}_{\text {thresh }}$, are assigned the same subcarrier modulation leading to a subcarrier throughput equal to $\log _{2}(M)$ bits/symbol. The SNR threshold, defined as the minimum subcarrier SNR which still guarantees BER $\leq$ $\mathrm{BER}_{\text {thresh }}$, on that specific subcarrier, is calculated as:

$$
\mathrm{SNR}_{\text {thresh }}=\frac{Q^{-1}\left(\frac{\mathrm{BER}_{\text {thresh }} \cdot \log _{2}(M)}{N_{b}}\right)^{2}}{\frac{d_{\min }^{2}}{2}}
$$

This concept is graphically explained in Fig. 3. For spatial subchannel 1, when using transmission blocking 4QAM subcarrier modulation, four subcarriers are unused because their subcarrier SNR is lower than $\mathrm{SNR}_{\text {thresh }}$ (4QAM), as yellowmarked. This implies that, for further calculations, the SNR and BER on these four unused subcarriers are not taken into account and that the throughput on these subcarriers is equal to 0 bits/symbol. Additionally, the throughput on all active subcarriers, marked with purple, is equal to 2 bits/symbol. Logically, when employing transmission blocking 16QAM subcarrier modulation, leading to a higher SNR threshold equal to $\mathrm{SNR}_{\text {thresh }}(16 \mathrm{QAM})$, less subcarriers are used for the same transmit power, as marked in blue for both spatial subchannels. Moreover, for transmission blocking 64 -and 256QAM subcarrier modulation, only six and two subcarriers are used for spatial subchannel 1, respectively, and no subcarriers are used for spatial subchannel 2 .

Also note the difference between a quasi-frequency-flat channel, as spatial subchannel 2, and a frequency-selective wideband channel, as spatial subchannel 1, typically occuring due to body-shadowing effects. For the quasi-frequency-flat wideband channel, only a small increase in SNR, visualized by the dotted blue line, could bring all subcarriers above, for example, $\mathrm{SNR}_{\text {thresh }}(16 \mathrm{QAM})$. When applying the same increase in SNR for the frequency-selective wideband channel, some subcarriers, which experience deep fading dips, remain under $\mathrm{SNR}_{\text {thresh }}(16 \mathrm{QAM})$. This implies that less subcarriers are used and, hence, that the mean throughput, defined as $\overline{\mathrm{thr}}$ and calculated by means of Formula (8), increases slower with increasing mean SNR.

$$
\overline{\operatorname{thr}}=\frac{1}{L} \sum_{l=1}^{L}\left(\frac{1}{r} \sum_{i=1}^{r}\left(\frac{1}{N} \sum_{k=1}^{N} \operatorname{thr}_{l, i, k}\right)\right)
$$

\section{B. Transmission blocking adaptive subcarrier modulation}

When applying transmission blocking adaptive subcarrier modulation, the modulation per subcarrier is not fixed but changes in an adaptive manner whether or not the subcarrier SNR is above the $\mathrm{SNR}_{\mathrm{thr}}$. More specifically, the highest possible modulation order, still guaranteeing a BER $\leq$ 

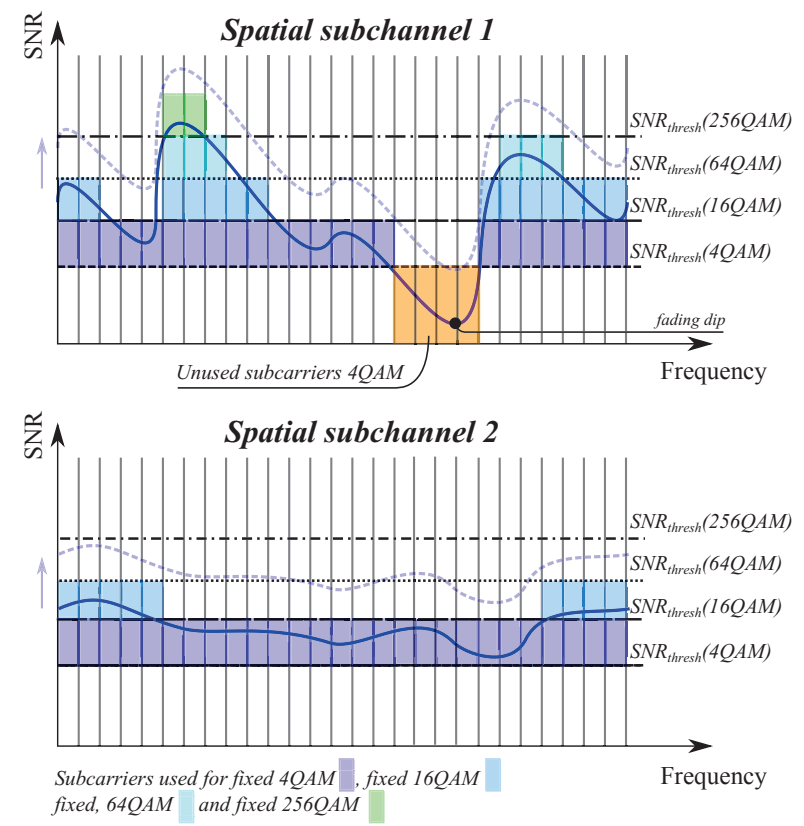

Fig. 3. Concept of transmission blocking fixed subcarrier modulation

$\mathrm{BER}_{\text {thresh }}$, is chosen per subcarrier. This concept is graphically explained in Fig. 4 which shows the different subcarrier modulations, depending on the subcarriers SNR's. As an example, for spatial subchannel 1, the subcarrier SNR is above the $\mathrm{SNR}_{\text {thresh }}(256 \mathrm{QAM})$ for two subcarriers, which implies that the throughput on these subcarriers is equal to 8 bits/symbol, while the BER $\leq \mathrm{BER}_{\text {thresh. }}$. Also note that, when $\mathrm{SNR}<$ $\mathrm{SNR}_{\text {thresh }}(4 \mathrm{QAM})$, the subcarriers are not used. For spatial subchannel 2, the subcarrier SNR is above $\operatorname{SNR}_{\text {thresh }}(16 \mathrm{QAM})$ for nine subcarriers which implies that the throughput on these subcarriers is 4 bits/symbol, while the BER $\leq \mathrm{BER}_{\text {thresh }}$.

\section{RESUlts}

Fig. 5 presents the BER performance of the four modulations, supported by IEEE 802.11 ac, when applying transmission blocking fixed subcarrier modulation, indicated by the different blue markers, with $\mathrm{BER}_{\text {thresh }}$ equal to $10^{-3}$. Additionally, the BER performance for the transmission blocking adaptive subcarrier modulation with $\mathrm{BER}_{\text {thresh }}$ equal to $10^{-3}$, is visualized by the pink markers. Beyond point A, the SNR of all subcarriers belonging to the strongest, quasi-frequency-flat channel is above $\mathrm{SNR}_{\text {thresh }}(4 \mathrm{QAM})$. Moreover, the strongest subcarriers of the weakest, frequency-selective spatial subchannel start exceeding $\mathrm{SNR}_{\text {thresh }}(4 \mathrm{QAM})$ and, hence, start contributing to the $\overline{\mathrm{BER}}$ and $\overline{\mathrm{SNR}}$. However, the SNR of these strongest subcarriers is only a few $\mathrm{dB}$ above $\mathrm{SNR}_{\text {thresh }}(4 \mathrm{QAM})$, which leads to a relatively high $B E R$, still below $B \mathrm{R}_{\text {thresh }}$, causing the flattening of the BER curve. At point B, almost all subcarriers on both spatial subchannels are 4QAM modulated. The effect of transmission blocking adaptive subcarrier modulation is clearly visualized by the pink markers. Between point $\mathrm{C}$ and $\mathrm{D}$, the subcarrier modulation switches from 4QAM to 256QAM owing
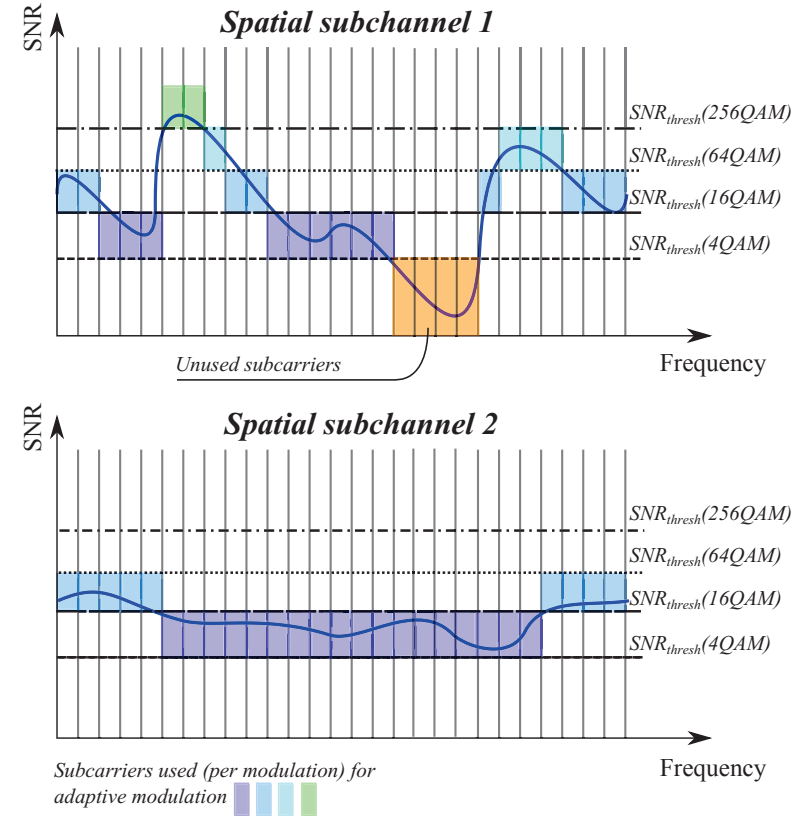

Fig. 4. Concept of transmission blocking adaptive subcarrier modulation

to increasing $\overline{\mathrm{SNR}}$. In this interval, the throughput increases, within the same order of BER magnitude, by switching to higher order modulations. At point $\mathrm{C}$, the majority of subcarriers are still 4QAM modulated whereas at point D, the majority of the subcarrier are 256QAM modulated.

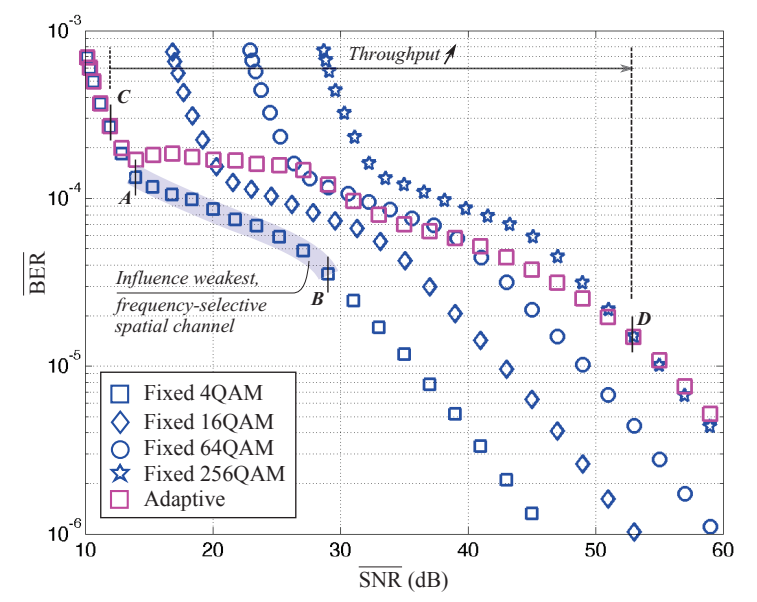

Fig. 5. Mean BER performance for all four modulations, supported by IEEE $802.11 \mathrm{ac}$, when applying transmission blocking fixed subcarrier modulation and mean BER performance when applying transmission blocking adaptive subcarrier modulation for a BER $\leq 10^{-3}$

Fig. 6 shows that the mean throughput, when applying transmission blocking adaptive subcarrier modulation is always higher than, or equal to, the scenario where transmission blocking fixed subcarrier modulation is applied. For low $\overline{\mathrm{SNR}}$, the adaptive modulation performs the same as fixed 4QAM because no subcarrier SNR is above $\mathrm{SNR}_{\text {thresh }}(16 \mathrm{QAM})$ and, hence, all active subcarriers are 4QAM modulated. Moreover, 
the adaptive modulation performs better than fixed 16-, 64and 256QAM because, for these higher order modulations, the subcarrier SNR do not exceed the corresponding threshold to ensure $\mathrm{BER} \leq \mathrm{BER}_{\text {thresh }}$. This implies that transmission on all subcarriers is blocked, leading to a mean throughput equal to 0 bits/symbol. When increasing $\overline{\mathrm{SNR}}$, some subcarriers, which are fixed to 4QAM for transmission blocking fixed subcarrier modulation, switch to 16QAM for transmission blocking adaptive subcarrier modulation, which leads to a higher $\overline{\mathrm{thr}}$ for adaptive subcarrier modulation. When focussing on fixed 256QAM, the throughput of adaptive subcarrier modulation is higher, for $\overline{\mathrm{SNR}} \leq 60 \mathrm{~dB}$, because much more subcarriers are used with a lower modulation order, leading to a higher $\overline{\mathrm{thr}}$.

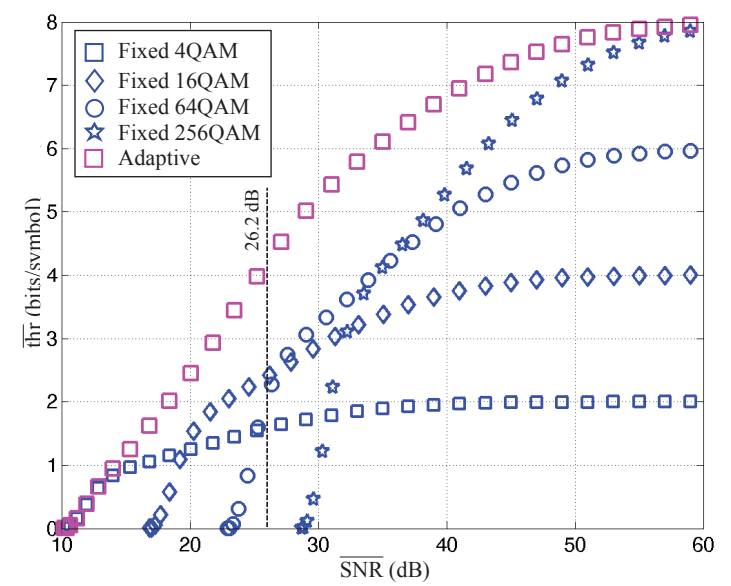

Fig. 6. Mean throughput for all four modulations, supported by IEEE 802.11 ac, when applying transmission blocking fixed subcarrier modulation and mean throughput when applying transmission blocking adaptive subcarrier modulation for a BER $\leq 10^{-3}$

From a practical point of view, when the transmit power of the $2 \times 2$ MIMO-OFDM system is equal to only $1.5 \mathrm{~mW}$, the received SNR on all four SISO links is high enough to ensure reliable timing synchronization and frequency offset estimation. This leads to a received mean SNR equal to $26.2 \mathrm{~dB}$, corresponding to the mean throughputs presented in Table I. The total bitrate $R_{b}$, in bps, for the a MIMO-OFDM system with transmission blocking is calculated as:

$$
R_{b}=r \cdot N \cdot \frac{\overline{\mathrm{thr}}}{T_{s}}
$$

Table I also presents the bitrate, for BER thresh equal to $10^{-3}$, when assuming $2 \times 2$ MIMO-OFDM with $80 \mathrm{MHz}$ OFDM bandwidth, leading to $r=2$ spatial subchannels and $\mathrm{N}=256$ subcarriers. Moreover, the IEEE 802.11 ac standard defines $T_{s}$ equal to $3.6 \mu \mathrm{s}$. Table I clearly shows that the mean throughput, and hence, the total bitrate can drastically increase when employing adaptive subcarrier modulation. The minimal bitrate increase is equal to $261 \mathrm{Mbps}$ when comparing fixed 64QAM $\left(R_{b}=343 \mathrm{Mbps}\right)$ with adaptive subcarrier modulation ( $\left.R_{b}=604 \mathrm{Mbps}\right)$.
TABLE I

MEAN THROUGHPUT, IN BITS/SYMBOL, AND CORRESPONDING BITRATE, IN MBPS, WHEN USING 1.5 MW TRANSMIT POWER

\begin{tabular}{c|cccc|c} 
& 4QAM & 16QAM & 64QAM & 256QAM & Adaptive \\
\hline$\overline{\text { thr }}$ & 1.59 & 2.41 & 2.17 & 0 & 4.25 \\
$R_{b}$ & 226 & 343 & 309 & 0 & 604
\end{tabular}

\section{CONCLUSion}

This paper shows that the throughput and, hence, data rate of a wideband indoor body-to-body channel is drastically increased when using transmission blocking adaptive subcarrier modulation compared to transmission blocking fixed subcarrier modulation. This allows on-duty firefighters to broadcast multimedia and real-time, on-body sensor data which increases their safety when operating in wideband public safety networks.

\section{ACKNOWLEDGMENT}

The authors would like to thank IAP BESTCOM and UCL for providing the Elektrobit channel sounder and measurement locations.

\section{REFERENCES}

[1] "LTE-Advanced Evolution in Releases 12 - 14," Nokia Solutions and Networks white paper, Tech. Rep., 2015.

[2] "Public Safety Mobile Broadband," Ericsson white paper, Tech. Rep., 2014.

[3] S. Lemey and H. Rogier, "SIW textile antennas as a novel technology for UWB RFID tags," in Proceedings of RFID Technology and Applications Conference (RFID-TA). Tampere, Finland: IEEE, September 2014, pp. 256-260.

[4] "802.11ac," IEEE Standard for Information technology - Telecommunications and information exchange between systems - Local and metropolitan area networks - Specific requirements - Part 11: Wireless LAN Medium Access Control (MAC) and Physical Layer (PHY) Specifications Amendment 4: Enhancements for Very High Throughput for Operation in Bands below $6 \mathrm{GHz}$, Tech. Rep., 2013.

[5] J. Faezah and K. Sabira, "Adaptive Modulation for OFDM systems," International Journal of Communication Networks and Information Security (IJCNIS), vol. 1, no. 2, August 2009.

[6] A. Czylwik, "Adaptive OFDM for wideband radio channels," in Global Telecommunications Conference Communications (GLOBECOM), vol. 1, 1996, pp. 713-718.

[7] T. Keller and L. Hanzo, "Adaptive modulation techniques for duplex ofdm transmission," IEEE Transactions on Vehicular Technology, vol. 49, no. 5, pp. 1893-1906, 2000.

[8] S. T. Chung and A. Goldsmith, "Degrees of freedom in adaptive modulation: a unified view," in 53rd Vehicular Technology Conference (VTC), vol. 2, 2001, pp. 1267-1271.

[9] J. Garzas, C. Calzon, and A. Armada, “An Energy-Efficient Adaptive Modulation Suitable for Wireless Sensor Networks with SER and Throughput Constraints," EURASIP Journal on Wireless Communications and Networking.

[10] M. Sudjai, L. Tran, and F. Safaei, "Adaptive space-time-frequency-coded UWB system for wireless body area network," EURASIP Journal on Wireless Communications and Networking.

[11] R. Janaswamy, Radiowave Propagation and Smart Antennas for Wireless Communications (The Springer International Series in Engineering and Computer Science). Springer, 2000.

[12] A. Goldsmith, Wireless Communications. Cambridge University Press, 2005.

[13] H. Suzuki, T. V. A. Tran, and I. B. Collings, "Characteristics of MIMOOFDM channels in indoor environments," EURASIP Journal on Wireless Communications and Networking, vol. 2007, no. 1, pp. 1-9, December 2006. 